\section{SOURCE-BOOK OF LABORATORY INFORMATION}

Laboratory Handbook

Edited by N. L. Parr. Pp. xi+1520. (London: George Newnes Ltd., 1963.) $210 s$.

$\mathrm{T}$ HIS is a remarkable book: within its 1520 pages of well-illustrated text, the editor and 46 other contributors have sought to provide " . . a useful source of information for those already engaged in any given field to technique and equipment employed elsewhere which may be relevant or capable of adaptation for their particular problems".

The editor has organized the book into twelve parts and in the first very briefly reviews laboratory design, organization and administration-mainly from the public service aspect. Part 2, construction of apparatus, includes, inter alia, chapters on electronics, remote-handling, furnaces and a particularly useful introduction to the manipulation of glass. The third part is concerned with measurement -dimensional, optical, electrical and thermal. Part 4 deals with essential techniques such as photography, optical and electron microscopy, X-ray crystallography and vacuum technology, while Part 5 covers the more specialist requirements, for example, low temperatures, distillation, high purity, inductive and dielectric heating; radioisotopes are given a whole part (6) to themselves. Part 7 contains 238 pages and is a comprehensive survey of chemical analysis. Particularly commendable is the chapter on physical methods which furnishes excellent introductions to the principles, instrumentation and procedures of emission spectrography, polarography, flame photometry, $\mathbf{X}$-ray diffraction and fluorescence techniques, electron microscopy and diffraction, radioactivation analysis and ultra-violet, visible and infra-red absorptiometry. Included are comprehensive tables of detection limits, diagnostic wave-lengths and half-wave potentials in a variety of base electrolytes. On the other hand, it is to be regretted that the editor was unable to include sections on such important aids to the analyst as mass spectrometry (dismissed in two lines in Part 5) and atomic absorption; instruments for both techniques are now commercially available. More understandable perhaps is omission from a book of this type of reference to specialist tools of the organic chemist, such as nuclear magnetic resonance, electron spin resonance and optical rotatory dispersion equipment.

Part 8 is equally valuable: five chapters (186 pages) cover mechanical testing, non-destructive testing, industrial radiography, metal corrosion and soil tests. Part 9 is concerned with the work and equipment of specialized laboratories : eighteen widely divergent fields are discussed (from forensic to pharmaceutical, rubber to refractory) and yet the editor apologizes for omissions (for example, forestry and building). Laboratory hazards, including fire, toxicity and radiation, are succinctly reviewed in Part 10, while three chapters in Part 11 briefty consider the recording, analysis and presentation of results. Here the 'non-specialist' is more likely to be deterred by the condensed account, if he is not already familiar with the 'simple statistical procedures' given: this is unfortunate since a wider appreciation of the value of analyses of variance and tests of significance is desirable-not to mention standardization of nomenclature. Finally, Part 12 indicates various information services and methods of publication. The general index contains less than 2,000 entries: in a book of this size and scope this might be considered barely adequate.

In a necessarily superficial work, it is not difficult to make minor criticisms within a given field. Thus there is a lack of correlation between references in various sections to electronic devices: the brief descriptions of primitive valve and transistor circuits in Chapters 3 and 6 serve only to whet the appetite. Only the non-chemist would find useful the summary tables of gravimetric and volumetric procedures in Chapter 32. No distinction is made between a.c. and d.c. polarography, although appropriate instruments are named. Frequent mention is made of 'colour temperature', but no cross-reference is given to the short explanation in Chapter 13 -and no mention at all in the index. The scientific application of ciné photography is briefly dismissed and the availability in Britain of magnetic stripe for 8-mm sound films is not mentioned. A few inevitable typographical errors have escaped notice, but they are mainly self-evident.

Notwithstanding these and similar criticisms, this handbook surely achieves the editor's primary aim While the price will generally preclude purchase by the individual, this work should not be buried in the laboratory central library; copies should be made available on a divisional basis for general browsing by staff at all levels.

G. F. Phincips

\section{STRUCTURE AND FUNCTION OF THE LIVER}

\section{The Liver}

Morphology, Biochemistry, Physiology. Edited by Ch. Rouiller. Vol. 1: Pp. xiii +683; 26 dollars. Vol. 2: Pp. xiv $+674 ; 171 s$. 6d. (New York: Academic Press, Inc.; London: Academic Press, Inc. (London), Ltd., 1963.)

HIS book in two volumes attemptsto correlatehepatic structure, function and biochemistry. Particular emphasis is laid on the subcellular and molecular levels as revealed by electron microscopy, histochemistry, cytochemistry and by isotopic methods. Many authors are concerned, all active workers in their respective fields, mainly derived from Switzerland and the United States with a few representatives from Britain, Canada, France, Germany and Hungary.

The editor, Ch. Rouiller, is an eminent electron microscopist, and this technique is particularly well covered, both in his two very comprehensivo chapters and in those of others. Guido Majoro contributes a thought-provoking chapter on liver coll death, and Stanley Hartroft an outstanding account of experimental cirrhosis. K. Alterman uses electron microscopy to describe sinusoids and sinusoidal cells. Hans Elias (p. 44) provides a valuable diagram of the ultrastructure of the liver cells, and A. M. Rappaport (p. 176), in another diagram, attempts to correlate the ultrestructure with the cellular metabolism.

The cyto- and histo-chemistry of the livor are discussed by M. Wachstein. The isolation, morphology and biochemistry of the cell components of the liver are described by Yvonne Moulé and Jean Chauveau. These authors advise caution in the interpretation of the results, for the cell fractions are not in the state they find themselves in situ and a mean value is obtained without reference to extremes in the population. Nevertheless, such methods do allow investigation of functional interrelationships within the coll. The importance of nuclear cytoplasmic intorrelationships in respect of DNA and RNA is omphasized.

A. M. Du Bois writes a fascinating account of the human embryonic liver. He touchos on normal anatomy and physiology, regretting that more is not known of bile secretion in the ombryo. The section on abnormalities is rather brief and will be of little help to the clinician anxious to know more of particular defoct in any individual patient.

H. Elias emphasizes the continuous nature of the hepatic parenchyma. Lobules are imaginary areas which can be created arbitrarily around portal canals or hepatic vein roots. A. M. Rappaport prefers to group the liver anatomically into acinar units arranged around an axis of terminal hepatic arterioles, portal venules and bile 